\title{
TOTAL DEGREE BOUNDS FOR ARTIN L-FUNCTIONS AND PARTIAL ZETA FUNCTIONS
}

\author{
Lei Fu And Daging Wan
}

\section{Introduction}

Let $\mathbf{F}_{q}$ be a finite field of characteristic $p$ with $q$ elements. Choose an algebraic closure $\mathbf{F}$ of $\mathbf{F}_{q}$. Throughout this paper, schemes, morphisms and sheaves defined over the base field $\mathbf{F}_{q}$ are denoted by letters with subscripts 0 . We indicate the base extension from $\mathbf{F}_{q}$ to $\mathbf{F}$ by dropping the subscripts 0 . Schemes and morphisms are separated and of finite type.

Let $X_{0}$ be a scheme over $\mathbf{F}_{q}$ and let $F: X \rightarrow X$ be the geometric Frobenius correspondence. For any endomorphism $f: X \rightarrow X$, denote by $\Lambda(f)$ the number of fixed points of $f$, that is,

$$
\Lambda(f)=\#\{x \in X(\mathbf{F}) \mid f(x)=x\} .
$$

Let $G$ be a finite group acting on $X_{0}$ on the right and let $\rho: G \rightarrow \operatorname{GL}(V)$ be a finite dimensional $\overline{\mathbf{Q}}_{l}$-representation of $G$, where $l$ is a prime number different from $p$. For each positive integer $k$, set

$$
v_{k}=\frac{1}{\# G} \sum_{g \in G} \operatorname{Tr}\left(\rho(g)^{-1}\right) \Lambda\left(g F^{k}\right) .
$$

The Artin $L$-function $L\left(X_{0}, \rho, t\right)$ is defined by

$$
L\left(X_{0}, \rho, t\right)=\exp \left(\sum_{k=1}^{\infty} v_{k} \frac{t^{k}}{k}\right) .
$$

By a well known theorem of Grothendieck $([\mathrm{G}])$, this is a rational function. Define its total degree totdeg $L\left(X_{0}, \rho, t\right)$ to be the sum of the number of its zeros and the number of its poles counted with multiplicities. In [BS], under the assumption that $X_{0}$ is a closed subscheme of the $n$-dimensional affine space $\mathbf{A}_{0}^{n}$ defined by the vanishing of polynomials of degrees at most $d$ and $G$ acts linearly on $X_{0}$ in the sense that $G \subset \mathrm{GL}\left(\mathbf{A}_{0}^{n}\right)$, Bombieri and Sperber prove that

$$
\operatorname{totdeg} L\left(X_{0}, \rho, t\right) \leq(\operatorname{dim} \rho)^{2}(4 d+9)^{4 n} .
$$

If the action of $G$ on $X_{0}$ is not linear, an explicit total degree bound can also be derived from the remark in [BS], but the resulting bound would depend on the order of the group $G$. As indicated in [BS], in some special cases, better

Received April 2, 2002.

Revised version received September 29, 2002. 
bounds are known and these improved bounds are of considerable importance in estimating character sums, because they lead to estimates independent of the order of $G$.

In this paper, we give a bound for totdeg $L\left(X_{0}, \rho, t\right)$ without assuming $G$ acts linearly on $X_{0}$. Our bound is independent of the order of $G$ and hence is important for estimating character sums. Precisely, we have

Theorem 0.1. Let $X_{0}$ be a closed subscheme of $\mathbf{A}_{0}^{n}$ defined by the vanishing of $r$ polynomials of degrees at most $d$. Let $G$ be a finite group acting on $X_{0}$ on the right and let $\rho: G \rightarrow \mathrm{GL}(V)$ be a finite dimensional $\overline{\mathbf{Q}}_{l}$-representation. Let $V=n_{1} V_{1} \oplus \cdots \oplus n_{k} V_{k}$ be the decomposition of $V$ into a direct sum of irreducible representations of $G$, where each $n_{j}$ is the multiplicity of the irreducible representation $V_{j}$ appeared in $V$. Then we have

$$
\operatorname{totdeg} L\left(X_{0}, \rho, t\right) \leq 3 \cdot 2^{r+1} \cdot(3+r d)^{n+1} \cdot\left(\sup _{j}\left\{\frac{n_{j}}{\operatorname{dim} V_{j}}\right\}\right) .
$$

Obviously we have $\sup _{j}\left\{\frac{n_{j}}{\operatorname{dimV} V_{j}}\right\} \leq \operatorname{dim} \rho$. So we have

Corollary. Notation as in Theorem 0.1. We have

$$
\operatorname{totdeg} L\left(X_{0}, \rho, t\right) \leq 3 \cdot 2^{r+1} \cdot(3+r d)^{n+1} \cdot(\operatorname{dim} \rho) .
$$

The main idea of our proof is to use Katz's bound on the sum of Betti numbers ([K1]) and a fixed point formula. Originally we only get the estimate in the Corollary. The estimate in Theorem 0.1 is suggested by the referee.

We now describe our second result. Let $X_{0}$ be a closed subscheme of $\mathbf{A}_{0}^{n}$ defined by the vanishing of some polynomials. Let $d_{1}, \ldots, d_{n}$ be $n$ positive integers and let $N_{d_{1} \ldots d_{n}}\left(k, X_{0}\right)$ be the number of points $\left(x_{1}, \ldots, x_{n}\right)$ in $X(\mathbf{F})$ such that $x_{1} \in \mathbf{F}_{q^{k d_{1}}}, \ldots, x_{n} \in \mathbf{F}_{q^{k d_{n}}}$. In [W1], Wan defines the partial zeta function $Z_{d_{1} \ldots d_{n}}\left(X_{0}, t\right)$ to be

$$
Z_{d_{1} \ldots d_{n}}\left(X_{0}, t\right)=\exp \left(\sum_{k=1}^{\infty} N_{d_{1}, \ldots d_{n}}\left(k, X_{0}\right) \frac{t^{k}}{k}\right) .
$$

He proves that $Z_{d_{1} \ldots d_{n}}\left(X_{0}, t\right)$ is rational if the integers $d_{i}$ 's can be arranged such that $d_{1}\left|d_{2}\right| \cdots \mid d_{n}$, generalizing Dwork's classical rationality theorem. In general, it is not clear if the partial zeta function is always rational, but Faltings [W1] noted that the partial zeta function is always nearly rational in the sense that there are some roots of unity $\lambda_{k}$ and some algebraic numbers $\mu_{k}(k=1, \ldots, m)$ such that

$$
Z_{d_{1} \ldots d_{n}}\left(X_{0}, t\right)=\prod_{k=1}^{m}\left(1-\mu_{k} t\right)^{\lambda_{k}} .
$$

In the above expression, by collecting similar terms, we may also assume that the $\mu_{k}(k=1, \ldots, m)$ are distinct, but then the $\lambda_{k}$ may not be roots of unity anymore. In this case, we define the total degree of $Z_{d_{1} \ldots d_{n}}\left(X_{0}, t\right)$ to be $\sum_{k=1}^{m}\left|\lambda_{k}\right|$. Here we fix an archimedean absolute value on the field of algebraic numbers. 
Theorem 0.2. Let $X_{0}$ be a closed subscheme of $\mathbf{A}_{0}^{n}$ defined by the vanishing of $r$ polynomials of degrees at most $d$. Let $d_{1}, \ldots, d_{n}$ be $n$ positive integers and let $m$ be their least common multiple. Then we have

$$
\operatorname{totdeg} Z_{d_{1} \ldots d_{n}}\left(X_{0}, t\right) \leq 3 \cdot 2^{m r+1} \cdot(3+m r d)^{d_{1}+\cdots+d_{n}+1} .
$$

The main idea of the proof of Theorem 0.2 is again to use Katz's bound on the sum of Betti numbers, an observation of Faltings, and a fixed point formula. As an immediate application of Theorem 0.2 , one obtains an explicit total degree bound for the geometric moment zeta functions [W5] (which are rational functions) attached to a family of algebraic varieties over finite fields. In particular, one obtains explicit estimates for the constants in the higher degree excess theorems in Katz [K2] in his statistical study of the universal family of smooth projective hypersurfaces over finite fields. In some special cases, Theorem 0.2 can be greatly improved, especially about the asymptotic behavior of the bound as $m$ goes to infinity while some of the $d_{i}$ 's are fixed. The universal family of elliptic curve and its relations to dimensions of modular forms of high weights provide such an example. Another interesting case to consider is the higher moment zeta function arising from the universal family of smooth projective hypersurfaces.

As an archimedean analogue of Dwork's $p$-adic unit root zeta functions, certain pure weight L-functions arising from a family of algebraic varieties were introduced in [W2]. The rationality of these pure weight L-functions depends on the full strength of Deligne's main theorem [D] which says that the $l$-adic higher direct image sheaves with compact support are mixed. We do not yet know how to give an explicit total degree bound for these deeper pure weight L-functions. The "degree bound" problem, that is, the lower bound problem for the $p$-adic Newton polygon, for the pure ( $p$-adic) slope L-functions in Dwork's original conjecture is even more difficult, as these pure slope L-functions [W3][W4] are $p$-adic meromorphic functions and in general not rational any more. In the so-called rank one case, an explicit lower bound for the p-adic Newton polygon has been worked out in [W4]. No non-trivial bound is known in the higher rank case.

Although our principal motivation of this paper comes from theoretical considerations, we have also been influenced by practical algorithmic computations of zeta functions and L-functions. Our explicit bounds are useful in explicitly computing the related zeta functions and L-functions. This is an emerging new subject, which we shall not discuss it here, but see [W6] for an expository introduction.

\section{Proof of the Theorems}

We need the following result of Katz ([K1], Corollary of Theorem 1): 
Proposition 1.1 (Katz). Let $X$ be a closed subscheme of $\mathbf{A}^{n}$ over $\mathbf{F}$ defined by the vanishing of $r$ polynomials of degrees at most $d$. We have

$$
\sum_{i=0}^{2 \operatorname{dim} X} \operatorname{dim} H_{c}^{i}\left(X, \overline{\mathbf{Q}}_{l}\right) \leq 3 \cdot 2^{r+1} \cdot(3+r d)^{n+1} .
$$

We also need the following fixed point formula:

Proposition 1.2. Let $X_{0}$ be a quasi-projective scheme over $\mathbf{F}_{q}$. For any $\mathbf{F}_{q^{-}}$ automorphism $\sigma_{0}: X_{0} \rightarrow X_{0}$ of finite order and for any positive integer $k$, we have

$$
\Lambda\left(\sigma F^{k}\right)=\sum_{i=0}^{2 \operatorname{dim} X}(-1)^{i} \operatorname{Tr}\left(\sigma F^{k}, H_{c}^{i}\left(X, \overline{\mathbf{Q}}_{l}\right)\right),
$$

where $F: X \rightarrow X$ is the geometric Frobenius correspondence and $\Lambda\left(\sigma F^{k}\right)$ is the number of fixed points of $\sigma F^{k}: X \rightarrow X$.

To prove this fixed point formula, we use Galois decent theory to show that there exists another scheme $X_{0}^{\prime}$ over $\mathbf{F}_{q}$ such that $X^{\prime}$ is isomorphic to $X$ over $\mathbf{F}$ and the geometric Frobenius correspondence on $X^{\prime}$ coincides with $\sigma F$ on $X$. Proposition 1.2 for the case $k=1$ then follows from the fixed point formula for the geometric Frobenius correspondence on $X^{\prime}$. The general case can be proved by working over the base $\mathbf{F}_{q^{k}}$.

Another result we need is the following well-known fact:

Lemma 1.3. Let $G$ be a finite group, $\rho: G \rightarrow G L(V)$ a finite dimensional $\overline{\mathbf{Q}}_{l^{-}}$representation, and $F: V \rightarrow V$ a $G$-equivariant endomorphism of $V$. Then we have

$$
\operatorname{Tr}\left(F, V^{G}\right)=\frac{1}{\# G} \sum_{g \in G} \operatorname{Tr}(g F, V) .
$$

Proof. Note that since $F$ is $G$-equivariant, the subspace $V^{G}$ is invariant under the action of $F$. So we may talk about $\operatorname{Tr}\left(F, V^{G}\right)$. Consider the homomorphism

$$
\pi: V \rightarrow V, x \mapsto \frac{1}{\# G} \sum_{g \in G} g x .
$$

Then we have $\operatorname{im}(\pi)=V^{G}$ and $\left.\pi\right|_{V^{G}}=$ id. So we have

$$
\operatorname{Tr}\left(F, V^{G}\right)=\operatorname{Tr}\left(\pi F, V^{G}\right)=\operatorname{Tr}(\pi F, V)=\frac{1}{\# G} \sum_{g \in G} \operatorname{Tr}(g F, V) .
$$

Let's prove Theorem 0.1. Let $G$ be a finite group acting on the right of a quasi-projective scheme $X_{0}$ over $\mathbf{F}_{q}$, let $\rho: G \rightarrow \mathrm{GL}(V)$ be a finite dimensional $\overline{\mathbf{Q}}_{l}$-representation of $G$ and let

$$
L\left(X_{0}, \rho, t\right)=\exp \left(\sum_{k=1}^{\infty} v_{k} \frac{t^{k}}{k}\right)
$$


be the Artin $L$-function, where

$$
v_{k}=\frac{1}{\# G} \sum_{g \in G} \operatorname{Tr}\left(g^{-1}, V\right) \Lambda\left(g F^{k}\right)
$$

By Proposition 1.2, we have

$$
\begin{aligned}
v_{k} & =\frac{1}{\# G} \sum_{g \in G} \operatorname{Tr}\left(g^{-1}, V\right) \Lambda\left(g F^{k}\right) \\
& =\frac{1}{\# G} \sum_{g \in G} \operatorname{Tr}\left(g^{-1}, V\right) \sum_{i=0}^{2 \operatorname{dim} X}(-1)^{i} \operatorname{Tr}\left(g F^{k}, H_{c}^{i}\left(X, \overline{\mathbf{Q}}_{l}\right)\right) \\
& =\sum_{i=0}^{2 \operatorname{dim} X}(-1)^{i} \frac{1}{\# G} \sum_{g \in G} \operatorname{Tr}\left(g F^{k}, H_{c}^{i}\left(X, \overline{\mathbf{Q}}_{l}\right) \otimes_{\overline{\mathbf{Q}}_{l}} V^{*}\right),
\end{aligned}
$$

where $V^{*}$ denotes the representation of $G$ dual to $V$ and $F$ acts trivially on $V^{*}$. Combining with Lemma 1.3 applied to the representation $H_{c}^{i}\left(X, \overline{\mathbf{Q}}_{l}\right) \otimes \overline{\mathbf{Q}}_{l} V^{*}$ of $G$ and the equivariant endomorphism $F^{k}$, we get

$$
v_{k}=\sum_{i=0}^{2 \operatorname{dim} X}(-1)^{i} \operatorname{Tr}\left(F^{k},\left(H_{c}^{i}\left(X, \overline{\mathbf{Q}}_{l}\right) \otimes_{\overline{\mathbf{Q}}_{l}} V^{*}\right)^{G}\right) .
$$

So we have

$$
L\left(X_{0}, \rho, t\right)=\exp \left(\sum_{k=1}^{\infty} v_{k} \frac{t^{k}}{k}\right)=\prod_{i=0}^{2 \operatorname{dim} X} \operatorname{det}\left(I-F t,\left(H_{c}^{i}\left(X, \overline{\mathbf{Q}}_{l}\right) \otimes_{\overline{\mathbf{Q}}_{l}} V^{*}\right)^{G}\right)^{(-1)^{i+1}} .
$$

Therefore

$$
\operatorname{totdeg} L\left(X_{0}, \rho, t\right) \leq \sum_{i=0}^{2 \operatorname{dim} X} \operatorname{dim}\left(H_{c}^{i}\left(X, \overline{\mathbf{Q}}_{l}\right) \otimes_{\overline{\mathbf{Q}}_{l}} V^{*}\right)^{G} .
$$

Let $V=n_{1} V_{1} \oplus \cdots \oplus n_{k} V_{k}$ be the decomposition of $V$ into a direct sum of irreducible representations of $G$, where each $n_{j}$ is the multiplicity of the irreducible representation $V_{j}$ appeared in $V$. Then we have

$$
\operatorname{dim}\left(H_{c}^{i}\left(X, \overline{\mathbf{Q}}_{l}\right) \otimes \overline{\mathbf{Q}}_{l} V^{*}\right)^{G}=\sum_{j=1}^{k} n_{j} \operatorname{dim}\left(H_{c}^{i}\left(X, \overline{\mathbf{Q}}_{l}\right) \otimes_{\overline{\mathbf{Q}}_{l}} V_{j}^{*}\right)^{G}=\sum_{j=1}^{k} n_{j} m_{j},
$$

where $m_{j}=\operatorname{dim}\left(H_{c}^{i}\left(X, \overline{\mathbf{Q}}_{l}\right) \otimes_{\overline{\mathbf{Q}}_{l}} V_{j}^{*}\right)^{G}$. Note that $m_{j}$ equals the multiplicity of $V_{j}$ appeared in the representation $H_{c}^{i}\left(X, \overline{\mathbf{Q}}_{l}\right)$ of $G$. So we have $\sum_{j=1}^{k} m_{j} \operatorname{dim} V_{j} \leq$ 
$\operatorname{dim} H_{c}^{i}\left(X, \overline{\mathbf{Q}}_{l}\right)$. Therefore

$$
\begin{aligned}
\operatorname{dim}\left(H_{c}^{i}\left(X, \overline{\mathbf{Q}}_{l}\right) \otimes_{\overline{\mathbf{Q}}_{l}} V^{*}\right)^{G} & =\sum_{j=1}^{k} \frac{n_{j}}{\operatorname{dim} V_{j}} m_{j} \operatorname{dim} V_{j} \\
& \leq\left(\sup _{j}\left\{\frac{n_{j}}{\operatorname{dim} V_{j}}\right\}\right)\left(\sum_{j=1}^{k} m_{j} \operatorname{dim} V_{j}\right) \\
& \leq\left(\sup _{j}\left\{\frac{n_{j}}{\operatorname{dim} V_{j}}\right\}\right)\left(\operatorname{dim} H_{c}^{i}\left(X, \overline{\mathbf{Q}}_{l}\right)\right),
\end{aligned}
$$

and hence

$$
\operatorname{totdeg} L\left(X_{0}, \rho, t\right) \leq\left(\sup _{j}\left\{\frac{n_{j}}{\operatorname{dim} V_{j}}\right\}\right)\left(\sum_{i=0}^{2 \operatorname{dim} X} \operatorname{dim} H_{c}^{i}\left(X, \overline{\mathbf{Q}}_{l}\right)\right) .
$$

Theorem 0.1 then follows from Katz's bound in Proposition 1.1.

Now we turn to the proof of Theorem 0.2. Let $X_{0}$ be a closed subscheme of $\mathbf{A}_{0}^{n}$ over $\mathbf{F}_{q}$ defined by the vanishing of $r$ polynomials of degrees at most $d$. Let $d_{1}, \ldots, d_{n}$ be $n$ positive integers and let $m$ be their least common multiple. Let $Y_{0}$ be the subvariety of the $m$-fold product $X_{0}^{m}$ defined as follows: For any point $\left(x_{1}, \ldots, x_{m}\right)$ in $X^{m}(\mathbf{F})$, where each $x_{j}$ is a point in $X(\mathbf{F}) \subset \mathbf{A}^{n}(\mathbf{F})$, write $x_{j}=\left(x_{1 j}, \ldots, x_{n j}\right)$. We define $Y_{0}$ to be the closed subvariety of $X_{0}^{m} \subset \mathbf{A}_{0}^{m n}$ defined by the equations

$$
x_{i j}=x_{i j^{\prime}} \text { whenever } j \equiv j^{\prime} \bmod d_{i}\left(i \in\{1, \ldots, n\}, j, j^{\prime} \in\{1, \ldots, m\}\right) .
$$

Keeping only the variables $x_{i j}$ with $1 \leq j \leq d_{i}(1 \leq i \leq n)$, one obtains a closed subvariety $Y_{0}^{\prime} \subset \mathbf{A}_{0}^{d_{1}+\cdots+d_{n}}$ defined by $m r$ equations of degrees at most $d$, and $Y_{0}^{\prime}$ is isomorphic to $Y_{0}$. Let $\sigma_{0}: X_{0}^{d} \rightarrow X_{0}^{d}$ be the automorphism defined by

$$
\left(x_{1}, \ldots, x_{d}\right) \rightarrow\left(x_{d}, x_{1}, \ldots, x_{d-1}\right) .
$$

Then $Y_{0}$ is invariant under the action of $\sigma_{0}$. For each positive integer $k$, as Faltings observed, one can show that

$$
N_{d_{1} \ldots d_{n}}\left(k, X_{0}\right)=\Lambda\left(\sigma F^{k}\right),
$$

where the left-hand side is the number of points $\left(x_{1}, \ldots, x_{n}\right)$ in $X(\mathbf{F})$ such that $x_{1} \in \mathbf{F}_{q^{k d_{1}}}, \ldots, x_{n} \in \mathbf{F}_{q^{k d_{n}}}$, and the right-hand side is the number of fixed points of the endomorphism $\sigma F^{k}: Y \rightarrow Y$. (Confer $\S 3$ in [W1]). For each $i$, let $\lambda_{i 1}, \ldots, \lambda_{i k_{i}}$ be all the distinct eigenvalues of $\sigma$ acting on $H_{c}^{i}\left(Y, \overline{\mathbf{Q}}_{l}\right)$ and let $H_{i 1}, \ldots, H_{i k_{i}}$ be the corresponding eigenvector spaces. Since $\sigma$ has finite order, each $\lambda_{i j}$ is a root of unity and

$$
H_{c}^{i}\left(Y, \overline{\mathbf{Q}}_{l}\right)=H_{i 1} \oplus \cdots \oplus H_{i k_{i}} .
$$

Since $F$ commutes with $\sigma$, each $H_{i j}$ is invariant under the action of $F$. Let $\mu_{i j 1}, \ldots, \mu_{i j k_{i j}}$ be all the eigenvalues of $F$ acting on $H_{i j}$, where $k_{i j}=\operatorname{dim} H_{i j}$. 
Each $\mu_{i j l}$ is an algebraic number. We have

$$
\operatorname{Tr}\left(\sigma F^{k}, H_{c}^{i}\left(Y, \overline{\mathbf{Q}}_{l}\right)\right)=\sum_{j=1}^{k_{i}} \sum_{l=1}^{k_{i j}} \lambda_{i j} \mu_{i j l}^{k} .
$$

By Proposition 1.2, we have

$$
\begin{aligned}
N_{d_{1} \ldots d_{n}}(k, X)=\Lambda\left(\sigma F^{k}\right) & =\sum_{i=0}^{2 \operatorname{dim} Y}(-1)^{i} \operatorname{Tr}\left(\sigma F^{k}, H_{c}^{i}\left(Y, \overline{\mathbf{Q}}_{l}\right)\right) \\
& =\sum_{i=0}^{2 \operatorname{dim} Y} \sum_{j=1}^{k_{i}} \sum_{l=1}^{k_{i j}}(-1)^{i} \lambda_{i j} \mu_{i j l}^{k} .
\end{aligned}
$$

So

$$
\sum_{k=1}^{\infty} N_{d_{1} \ldots d_{n}}(k, X) t^{k}=\sum_{i=0}^{2 \operatorname{dim} Y} \sum_{j=1}^{k_{i}} \sum_{l=1}^{k_{i j}}(-1)^{i} \lambda_{i j} t \frac{d}{d t} \ln \left(1-\mu_{i j l} t\right)^{-1},
$$

that is,

$$
t \frac{d}{d t} \ln Z_{d_{1} \ldots d_{n}}\left(X_{0}, t\right)=\sum_{i=0}^{2 \operatorname{dim} Y} \sum_{j=1}^{k_{i}} \sum_{l=1}^{k_{i j}}(-1)^{i} \lambda_{i j} t \frac{d}{d t} \ln \left(1-\mu_{i j l} t\right)^{-1},
$$

where $Z_{d_{1} \ldots d_{n}}\left(X_{0}, t\right)$ is the partial zeta function. This implies that

$$
Z_{d_{1} \ldots d_{n}}\left(X_{0}, t\right)=\prod_{i=0}^{2 \operatorname{dim} Y} \prod_{j=1}^{k_{i}} \prod_{l=1}^{k_{i j}}\left(1-\mu_{i j l} t\right)^{(-1)^{i+1} \lambda_{i j}}
$$

and

$$
\begin{aligned}
\operatorname{totdeg} Z_{d_{1} \ldots d_{n}}\left(X_{0}, t\right) & \leq \sum_{i=0}^{2 \operatorname{dim} Y} \sum_{j=1}^{k_{i}} \sum_{l=1}^{k_{i j}}\left|(-1)^{i+1} \lambda_{i j}\right| \\
& =\sum_{i=0}^{2 \operatorname{dim} Y} \sum_{j=1}^{k_{i}} \sum_{l=1}^{k_{i j}} 1 \\
& =\sum_{i=0}^{2 \operatorname{dim} Y} \operatorname{dim} H_{c}^{i}\left(Y, \overline{\mathbf{Q}}_{l}\right) \\
& =\sum_{i=0}^{2 \operatorname{dim} Y} \operatorname{dim} H_{c}^{i}\left(Y^{\prime}, \overline{\mathbf{Q}}_{l}\right) .
\end{aligned}
$$

Theorem 0.2 follows from this last inequality, Proposition 1.1, and the fact that $Y_{0}^{\prime}$ is a closed subscheme of $\mathbf{A}^{d_{1}+\cdots+d_{n}}$ defined by the vanishing of $m r$ polynomials of degrees at most $d$. 


\section{Acknowledgements}

We thank the referee for suggesting the estimate in Theorem 0.1, and for his many other suggestions. The research of Lei Fu is supported by Qiushi Science \& Technologies Foundation and Project 973. The research of Daqing Wan is partially supported by the NSF (DMS 9970417) and the NNSF of China (10128103). We also thank the Morningside Center of Mathematics in Beijing, where the present work was carried out.

\section{References}

[BS] E. Bombieri, S. Sperber, On the Degree of Artin L-functions in characteristic p, C. R. Acad. Sci. Paris Sér. I Math. 306 (1988), 393-398.

[D] P. Deligne, La Conjecture de Weil. II, Inst. Hautes Études Sci. Publ. Math. No. 52 (1980), 137-252.

[G] A. Grothendieck, Formule de Lefschetz et rationalité des fonctions L, Séminaire Bourbaki, Vol. 9, Exp. No. 279, 41-55, Soc. Math. France, Paris, 1995.

[K1] N. Katz, Sums of Betti numbers in arbitrary characteristic, Finite Fields Appl. 7 (2001), 29-44.

[K2] - Frobenius-Schur indicator and the ubiquity of Brock-Granville quadratic excess, Finite Fields Appl. 7 (2001), 45-69.

[W1] D. Wan, Partial zeta functions of algebraic varieties over finite fields, Finite Fields Appl. 7 (2001), 238-251.

[W2] _ Pure L-functions from algebraic geometry over finite fields, Finite fields and applications (Augsburg, 1999), 437-461, Springer, Berlin, 2001.

[W3] _ Higher rank case of Dwork's conjecture, J. Amer. Math. Soc. 13 (2000), 807852.

[W4] _ Rank one case of Dwork's conjecture, J. Amer. Math. Soc. 13 (2000), 853-908.

[W5] - Moment zeta functions in arithmetic geometry, preprint.

[W6] _ Algorithmic zeta functions over finite fields, to appear in MSRI Algorithmic Number Theory Proceedings.

Institute of Mathematics, Nankai University, Tianjin, P. R. China.

E-mail address: leifu@nankai.edu.cn

Department of Mathematics, University of California, Irvine, CA 92697, U.S.A.

E-mail address: dwan@math.uci.edu 